\section{Patients with chronic kidney disease have poor perceived knowledge of treatment options}

Education about chronic kidney disease (CKD) is important to facilitate informed decision making in patients with this condition and can improve medical outcomes. Finkelstein et al. have shown, however, that a large proportion of patients with CKD perceive that they have limited knowledge of their disorder and feel they have no knowledge of the various treatment options for end-stage renal disease (ESRD).

This study examined 676 North American patients with stage 3-5 CKD (glomerular filtration rate $<60 \mathrm{ml} / \mathrm{min} / 1.73 \mathrm{~m}^{2}$ ) who completed a self-administered questionnaire to assess their knowledge of CKD and renal replacement therapies. On average, patients had been receiving nephrology care for a mean of 5.2 years before completing the questionnaire. Despite this fact, only $23 \%$ of patients reported having a great deal or extensive knowledge of their CKD and $35 \%$ reported having very limited or no knowledge about kidney disease.

With regard to renal replacement therapy, $35 \%$ of patients reported having no knowledge of any ESRD treatment modality. In addition, more than $50 \%$ of patients reported having no knowledge of continuous ambulatory peritoneal dialysis, automated peritoneal dialysis, or transplantation. African American patients perceived themselves as having less knowledge of ESRD therapies than did individuals of other ethnic backgrounds.

The authors suggest that the perceived lack of knowledge of renal replacement therapies in patients with CKD reflects problems in the education of patients by nephrologists rather than lack of referral of such patients to nephrologists.

Original article Finkelstein FO et al. (2008) Perceived knowledge among patients cared for by nephrologists about chronic kidney disease and end-stage renal disease therapies. Kidney Int 74: 1178-1184

\section{Serum alkaline phosphatase is a predictor of mortality in patients on hemodialysis}

Disturbances in serum levels of minerals and parathyroid hormone (PTH) occur in renal osteodystrophy and are associated with mortality in patients on maintenance hemodialysis; however, the predictive value of alkaline phosphatase, another marker of metabolic bone disease, has not been thoroughly investigated in this setting. Regidor and colleagues sought, therefore, to identify the relationship between serum alkaline phosphatase levels and both all-cause and cardiovascular mortality in patients on hemodialysis.

Data from a total of 73,960 US patients on hemodialysis were assessed. Serum alkaline phosphatase levels were measured and classified into eight categories (range $<60 \mathrm{U} / \mathrm{I}$ to $\geq 180 \mathrm{U} / \mathrm{l}$, in $20 \mathrm{U} / \mathrm{l}$ increments). Increased alkaline phosphatase levels were associated with an increased risk of death over the 3-year study period. After adjustment for confounding risk factors, patients with alkaline phosphatase levels above the upper limit of normal $(\geq 120 \mathrm{U} / \mathrm{l})$ had a hazard ratio for death of $1.25(95 \% \mathrm{Cl}$ 1.21-1.29; $P<0.001)$. The association between alkaline phosphatase and death was independent of other markers of metabolic bone disease and was robust across subgroups of ethnicity, gender and hepatitis $\mathrm{C}$ seropositivity.

Serum alkaline phosphatase levels could be a better marker of metabolic bone disease in patients on hemodialysis than serum PTH, as this enzyme stems from the bone itself, whereas PTH is derived from the parathyroid glands.

Original article Regidor DL et al. (2008) Serum alkaline phosphatase predicts mortality among maintenance hemodialysis patients. J Am Soc Nephrol 19: 2193-2203

\section{New assay to improve detection of genetic abnormalities in ADPKD}

Currently used base-pair screening methods fail to detect large DNA rearrangements responsible for autosomal dominant polycystic kidney disease (ADPKD). To address this problem, investigators, including those from the Consortium for Radiological Imaging Studies of PKD (CRISP), have developed a new genetic assay based on multiplex ligation-dependent probe amplification.

The assay, which provides results within 2 days, detected 25 previously identified deletions (18 in PKD1/TSC2, 6 in PKD1 and 1 in PKD2). Analysis of the CRISP and other populations by use of the assay revealed 7 new mutations associated with typical ADPKD (6 in 